\title{
ENHANCED LOAD BALANCING STRATEGY IN HETEROGENEOUS PEER-TO-PEER NETWORKS
}

\author{
${ }^{1}$ P. Murugesan and ${ }^{2} A$. Shanmugam \\ ${ }^{1}$ Department of Computer Applications, Bannari Amman Institute of Technology, \\ Sathyamangalam-638401, Tamil Nadu, India \\ ${ }^{2}$ Bannari Amman Institute of Technology, Sathyamangalam-638401, Tamil Nadu, India
}

Received 2012-12-24, Revised 2013-06-25; Accepted 2013-07-20

\begin{abstract}
Existing P2P systems provide a Duty Cycle Data Appropriation (DCDA) Technique allocate objects based on ranking among "peer nodes" in a method that result in various nodes containing times as numerous objects as the standard node. Further disparity might effect owing to non-uniform allocation of objects in the identifier break and a high amount of heterogeneity in objective loads and node capabilities. With that, a node's load might differ seriously over time as the system can be projected to practice incessant operations of objects. Introduced an algorithm for load balancing in such heterogeneous, active P2P systems. Foremost contributions are: (1) suggest a fully dispersed method to preserve the record of file access information. This information is employed to forecast the prospect file access frequencies and sustain the load allocation and redeployment operations; (2) Plan a novel load balancing algorithm, which obtains the file access record and peer heterogeneity properties to establish the load allocation. Enhanced Load Balancing Strategy in Heterogenous P2P Network (ELBSHN) algorithm engender the best load allocation resolution when a novel peer enters, it also be capable to vigorously achieve the load redeployment through system running time if congested peers emerged. In ELBSHN algorithm, no virtual servers are employed; consequently encompass less processing overhead on the exclusive routing metadata preservation. An experimental evaluation is conducted using the Heterogeneous peer networks data extracted from the large internet service providers to estimate the performance of the proposed enhanced load balancing strategy. Simulation results illustrate that in the features of quick onsets and exits of objects of extensively changeable load, algorithm attains load balancing for system utilizations as high as $90 \%$ while affecting only regarding $8 \%$ of the load that appears into the system.
\end{abstract}

Keywords: Heterogeneous P2P Networks, Peer Server Node, Load Balancing Algorithm, TopologicallyAware Data Duplication Method

\section{INTRODUCTION}

Peer-to-Peer (P2P) representation concerned numerous attentions from both business and educational societies owing to its hopeful possessions for instance probable load harmonizing, self-autonomous association and competent computer funds operation. It is normally supposed that the $\mathrm{P} 2 \mathrm{P}$ representation have fine load balancing presentation. An unnecessary load will basis for long file salvage latencies and damage the scheme taken as a whole presentation. Obviously, a well considered load balancing algorithm is in huge need. DHT indexing schemes suffer either query inefficiency or high maintenance cost. LIGhtweight Hash Tree (LIGHT) a query-efficient yet low-maintenance indexing scheme. LIGHT employs a tree summarization strategy for graceful distribution of its index structure (Tang et al., 2010).

Corresponding Author: P. Murugesan, Department of Computer Applications, Bannari Amman Institute of Technology, Sathyamangalam-638401, Tamil Nadu, India 
Numerous solutions have been planned to lecture to the load balancing crisis. Nevertheless, these all imagine that the scheme is stationary and most suppose that the IDs of both nodes and items are consistently dispersed. Salem et al. (2010), enlarge a novel logical method for the valuation of the outage prospect of supportive Decode-and-Forward (DF) Automatic-Repeat-Request (ARQ) communicating beneath packet-rate vanishing (fast vanishing or block vanishing) channels, wherever the channels hang about permanent inside every ARQ communication round, but modify separately from one round to another (Lee et al., 2010). A central pairing system and a dispersed pairing method are urbanized for efficient load balancing strategies (Yang et al., 2009). node and item identifiers are arbitrarily selected, then there is an imbalance factor in the number of items amassed at a node. Several algorithms have been planned formerly for the load balancing approach employed in standardized environment. For mixed environment, (Hsiao et al., 2011) presented load balancing technique.

The previous several years have seen the manifestation of a group of prearranged peer-to-peer schemes that near a dispersed concept. In such prearranged systems, a exclusive identifier is connected with every data item and every node in the system. The identifier break is divided amongst the nodes that shape the Peer-to-Peer (P2P) system and every node is accountable for storing all the items that are charted to an identifier in its section of the space. New fuzzy dynamic load balancing algorithm for homogenous distributed systems utilizes fuzzy logic with inaccurate load information, making load distribution decisions and maintaining overall system stability (Alakeel, 2012).

In hopeful Orthogonal Frequency Division Multiple Access (OFDMA) schemes, downlink signs creating from the similar Base Station (BS) are orthogonal, as those from diverse BSs obstruct with every other. As a result, Inter-Cell Intrusion (ICI) turns out to be main presentation deprivation factor. Chiefly, boundary users go through from cruel ICI as well as the intrinsic near-far crisis. To progress cell edging presentations and hold a more unbiased data rate amongst all users, incomplete Frequency Regain (PFR) and load-balancing strategies are examined in (Son et al., 2009).

The calculating authority of any dispersed system can be appreciated by permitting its component Computational rudiments (CEs), or nodes, to effort helpfully so that huge loads are owed amongst them in a reasonable and efficient mode. Any approach for load allocation amongst CEs is termed as Load Balancing (LB). An efficient LB strategy makes sure finest exercise of the dispersed resources whereby no $\mathrm{CE}$ ruins in an inactive state as any other $\mathrm{CE}$ is being employed.

\section{MATERIALS AND METHODS}

A novel load balancing algorithm is presented to resolve the problems rose in all existing works. Load balancing is attained by touching from greatly overloaded corporeal nodes to carelessly burdened physical nodes. Regard as a structure with $\mathrm{N}$ nodes. If:

- Intend a proficient, dispersed method to gather file access record information; information is employed to forecast the potential file access inclination

- Suggest a trouble-free and precise load allocation method. When a novel peer enters, the workload on the peer which possesses the zone it will unite is fine divided. The dimension of the zone novel peer possesses is dogged by its capability and the file access record

- Devised a low overhead load reorganization method. As the method remains running, the size of the region possesses by a peer might be attuned to reproduce the change of the workload uniqueness

\subsection{Enhanced Load Balancing Strategy in Heterogeneous P2p Networks}

In the prospect heterogeneous wireless networks with a diverse operation of universal nodes in the network, diverse nodes will contend for the similar resources for obtaining mobile services. This facade huge confronts to resource organization and organization. One serious tread to accomplish the finest resource procedure is for the networks to attach to the access nodes that present the finest conditions to them. The study (Hu et al., 2010) proposes a novel connection method in such a heterogeneous surroundings that aspires to supply the finest service to the networks on both uplink and downlink as reducing the intrusion stage.

Employ file access record information to estimate the outlook file access frequencies. Algorithm encloses two workings such as a enhanced load balance algorithm which is employed when a novel peer enters and a load relocation algorithm which can be employed when a peer leaves the structure or one peer turn out to be overloaded. The architecture diagram of the Enhanced Load Balancing Strategy in Heterogeneous P2P Networks is shown in Fig. 1. 


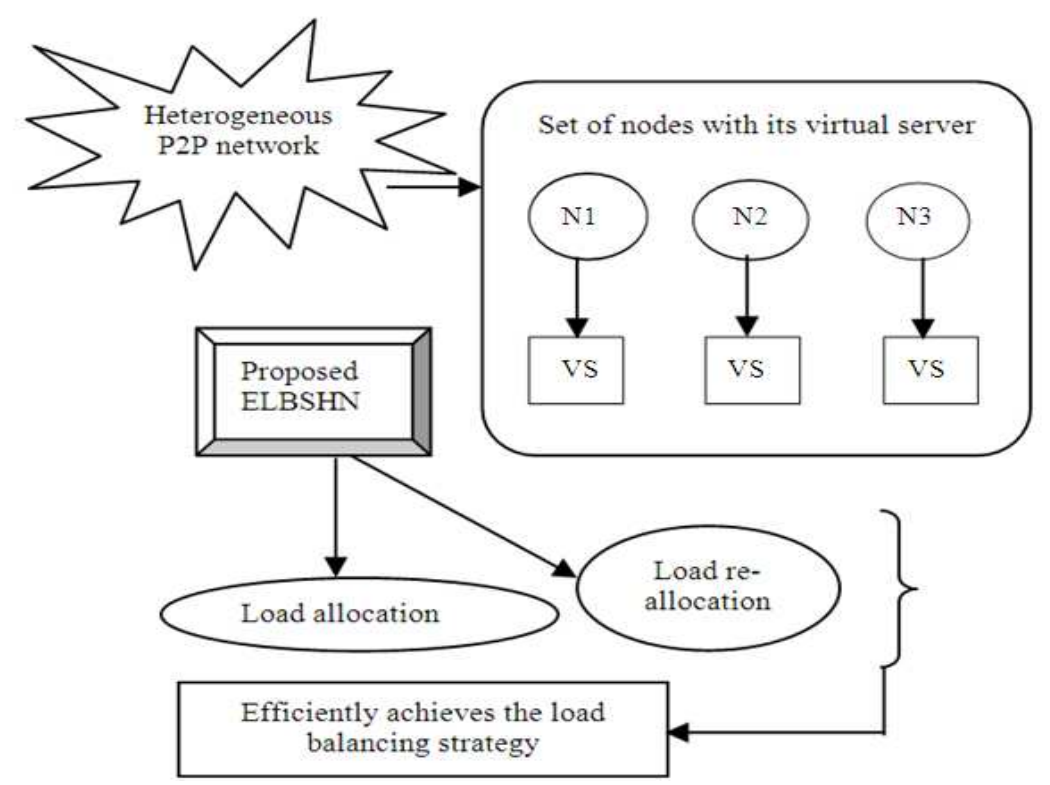

Fig. 1. Architecture diagram of the proposed ELBSHN

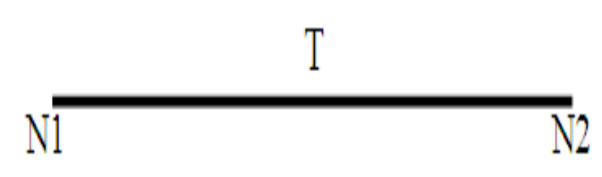

(a)

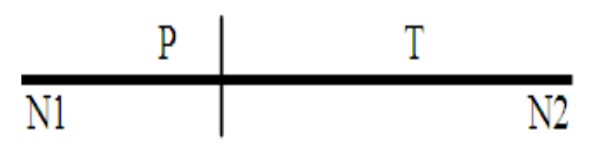

(b)

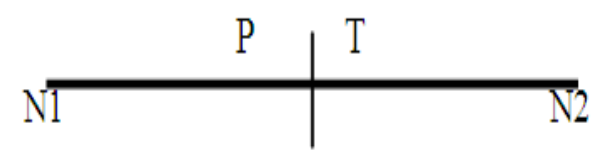

(c)

Fig. 2. Sample Peer Join Operation, (a) Before $P$ joins (b) Existing DCDA technique (c) Proposed ELBS

\subsection{Load Balancing Strategy}

When a novel peer $\mathrm{P}$ enters, the enhanced load allocation algorithm is implemented. The objective is to equalize the workload on node $\mathrm{P}$ and the peer $\mathrm{T}$ who possesses the zone $\mathrm{P}$ will unite. The separation of the workload formerly considered on $\mathrm{T}$ is dogged by numerous issues, for instance the capabilities of peers $T$ and $\mathrm{P}$, the projected future workload in the zone possessed by $\mathrm{T}$. In conventional DCDA systems, when a peer enters, a unique identifier (peerid) is allocated. In common, the peer's distinctive information for instance IP address can be employed as the starting point for this creation. Then the ranking terms are assigned and load diversion strategies are made.

For the novel peer $\mathrm{P}$, subsequent to this zone is established, it is not required to employ $p$ as the peerid. It decide one more location p' (inside this zone) which can well again partition the workload. To put into practice, in ELBSHN approach, both the chronological file access information and the capacities of these two peers are employed to construct the zone separation. Figure 2 shows an example. The novel peer $\mathrm{P}$ is specified the peerid p',other than the charge $\mathrm{p}$. Subsequent to the join procedure completed, $\mathrm{P}$ possesses the zone $(\mathrm{n} 1, \mathrm{p}$ '] and $\mathrm{T}$ possesses the zone (p',n2].

The peer join algorithm is shown in Fig. 3. Imagine the abilities for peers $\mathrm{P}$ and $\mathrm{T}$ are $\mathrm{CP}$ and CT. Prior to the peer join process is accomplished, for peer $\mathrm{T}$, the fields which are dropped in the zone $(\mathrm{n} 1, \mathrm{n} 2]$ are af1,af2,...,afe and the resultant admittance frequencies of these files are Faf1; Faf2; :::; Fafe. As a result, the combined workload in $(\mathrm{n} 1, \mathrm{n} 2)$ is:

$$
\mathrm{W}_{\mathrm{T}}=\mathrm{F}_{\mathrm{af} 1} * \mathrm{O}_{\mathrm{af} 1}+\ldots . .+\mathrm{F}_{\mathrm{afe}} \mathrm{O}_{\mathrm{afe}}=\sum_{\mathrm{i}=\mathrm{af1} 1}^{\mathrm{afe}} \mathrm{F}_{\mathrm{i}} \mathrm{O}_{\mathrm{i}}
$$




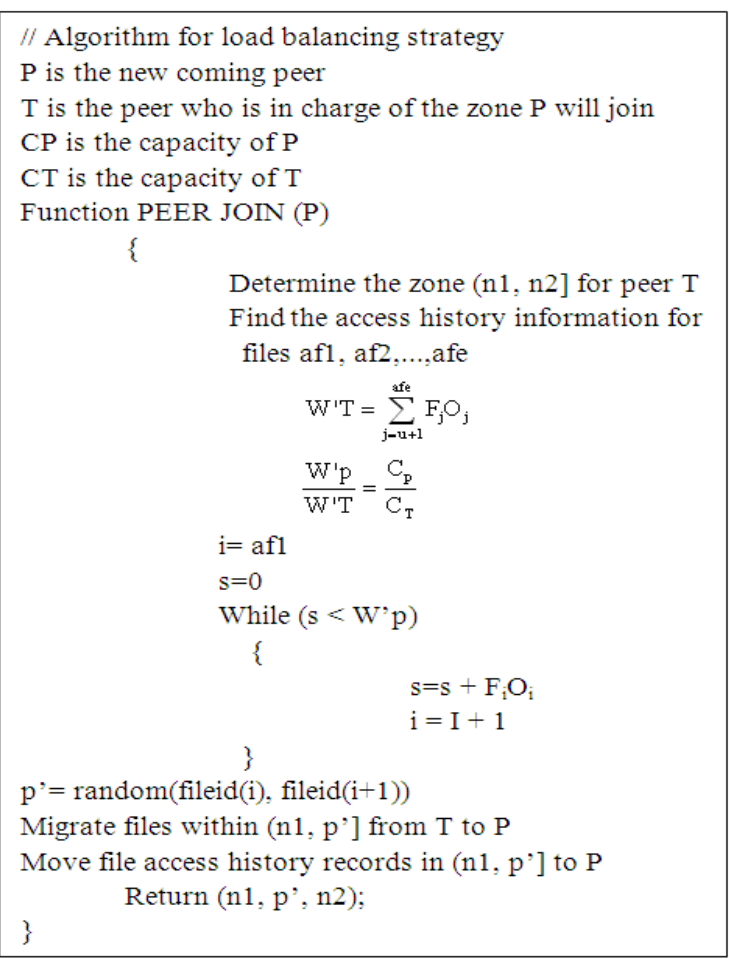

Fig. 3. Load balancing strategy

To fine separate WT, require discovering a number $\mathrm{u}$, such that af $1<\mathrm{u}<$ afe and contain:

$$
\frac{\mathrm{C}_{\mathrm{p}}}{\mathrm{C}_{\mathrm{T}}}=\frac{\sum_{\mathrm{i}=\mathrm{afl}}^{\mathrm{afe}} \mathrm{F}_{\mathrm{i}} \mathrm{O}_{\mathrm{i}}}{\sum_{\mathrm{j}=\mathrm{u}+1}^{\mathrm{a}} \mathrm{eF}_{\mathrm{i}} \mathrm{O}_{\mathrm{i}}}
$$

After this exacting value " $\mathrm{c}$ " is established, can arbitrarily prefer a location $\mathrm{p}^{\prime}$, such that $\mathrm{u}<\mathrm{p}$ ' $<(\mathrm{u}+1)$, as the peerid of the peer node $\mathrm{P}$, in place of $\mathrm{p}$. Since the prior workload WT on T is separated into the novel workloads W'P on P and W'T on T. Encompass the subsequent formulas:

$$
\mathrm{W}_{\mathrm{T}}=\mathrm{W}_{\mathrm{p}}^{\prime}+\mathrm{W}_{\mathrm{T}}^{\prime}
$$

Where: $W^{\prime} p=\sum_{i=a f 1}^{u} F_{i} O_{i}$

$$
W^{\prime} T=\sum_{j=u+1}^{\text {afe }} \mathrm{F}_{j} \mathrm{O}_{j}
$$

Thus: $\frac{W^{\prime} p}{W^{\prime} T}=\frac{C_{p}}{C_{T}}$

Then, the preceding workload WP is fine dispersed on peers $\mathrm{P}$ and $\mathrm{T}$. For the file admittance to be occurred in close to future, can wait for the workload should be capable to be fine owed to these two peers. If a peer foliages from the system, the zone formerly owed to it will be shared by means of the zone possessed by its descendant or antecedent. No additional process is desired. Nevertheless, such a approach might cause load disparity on that peer in the prospect. To alleviate this crisis, can separate the files formerly taken on the parting peer to its contiguous two peers.

\subsection{Load Redistribution}

In the exceeding peer join algorithm, region separation method is employed to guarantee the finest load division at the instance the new peer enters. Nevertheless, the file access frequencies forever transform from time to time. It is incredibly widespread that, after a definite quantity of time, a sizzling file with lofty access frequency develops into very frozen and no peers demand it any more. In difference, an initially bitter file might turn out to be very burning.

Alternatively, the workload reorganization engages prospect file access calculation and data association processes. It might initiate considerable quantity of overhead. Algorithm carried out load redeployment procedure only if it is essential. That is, one or two peers acquire congested. This could take place when a peer foliages the system and its descendant is congested or the alteration of file access behavior origin a peer congested slowly.

Figure 4 shows the load reorganization algorithm. Imagine the peer $\mathrm{K}$ is congested. It will establish a load relocation process. It associates its instantaneous descendant L1 and antecedent J1. Assume, their analogous workloads are WK, WL1 and WJ1, correspondingly. The combined capability of these three peers $A C=C K+C L 1+C J 1$. If the amount of workloads on $\mathrm{K}, \mathrm{L} 1$ and $\mathrm{J} 1$ is superior to $\mathrm{Vs} * \mathrm{AC}$, then more peers encompass to be concerned. Peer L1 will get in touch with its descendant L2 and peer J1 will contact its antecedent $\mathrm{J} 2$. If the combined workload AC $=\mathrm{CK}+\mathrm{CL} 1+\mathrm{CJ} 1+\mathrm{CL} 2+\mathrm{CJ} 2$ is at rest above $\mathrm{Vs} *$ $\mathrm{AC}$, more peers will be called. If the amount of the workloads is fewer than $\mathrm{Vs} * \mathrm{AC}$, the load reorganization process will be behaviored on the position of present peers. The workload inside these regions will be reorganized consistent with peers' abilities and the prospect file access inclinations. An inconsistency of the algorithm is, in every round, in its place of calculating a descendant and an antecedent; might comprise only one of them. Then, can decrease the quantity or commenced overhead. 


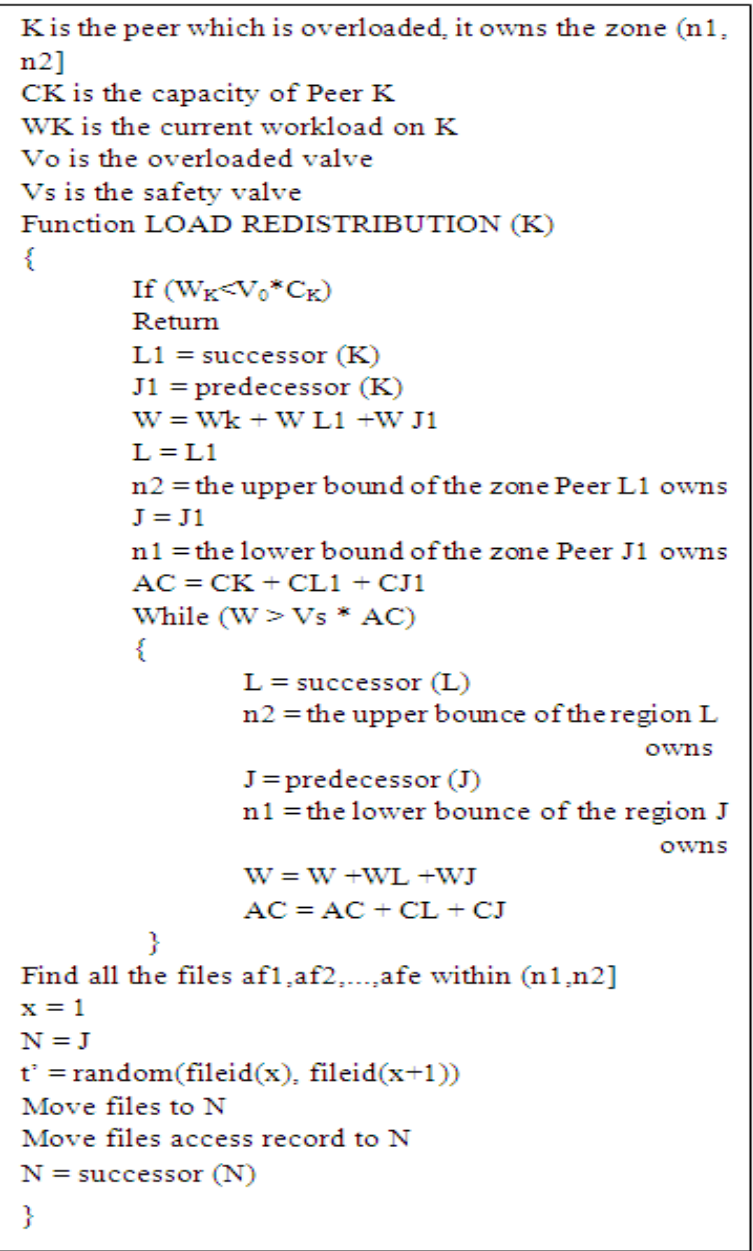

Fig. 4. Workload Redistribution Algorithm

\section{RESULTS AND DISCUSSION}

When compared to an existing works time variant peer heterogeneous P2P network using DTWA, heterogeneous peer node server cycle analyzer using DCDA technique and Node selection strategy, the Enhanced Load Balancing Strategy in Heterogeneous P2P Networks perform better by analyzing through the experimental evaluation. The below table and graph describes the performance of the ELBSHN. Initially, the simulation environment exercise in our experiments and then provide the simulation results. In our simulation, the holdups of intra-transit field links, stub-transit relations and intra-stub domain relations are locate to 100, 20 and $5 \mathrm{~ms}$ correspondingly. In all the research, the number of peers in replicated system is 5000. It differ the number of files in the scheme from $20 \mathrm{~K}$ to $100 \mathrm{~K}$. The ELBSHN is efficiently designed and implemented in NS-2 simulator.

In addition simulations are carried out to show the effectiveness of the ELBSHN work with bench mark data sets derived from UCI Repository. With the loaddemand factor obtained from the peer nodes processing abilities, peer servers processing cycle requirements are identified. Load diversions are made to peer server if the peer node gets overloaded. The diverse data nature of the command required by the peer servers is absolutely recognized. A priori of Data Format Load Points are coordinated to present data design insisting of particular nodes and its cause on load balancing the peer servers are considered. The performance of the ELBSHN is measured in terms of.

\subsection{Load Movement Factor}

Termed as the total association cost acquired owing to load balancing separated by the total cost of affecting all objects in the scheme once.

\subsection{Energy Consumption}

Amount of energy consumped in enhanced load balancing scheme for heterogeneous $\mathrm{P} 2 \mathrm{P}$ systems. It is measured in terms of percentage $(\%)$.

\subsection{Node Utilization}

Termed as the greatest in excess of all virtual times $t$ of the 99 percentile of the utilizations of the nodes at instance.

\subsection{Average overall execution time}

It is defined as the amount of time taken to perform the execution process. The overall execution process is measured in terms of seconds $(\mathrm{sec})$.

The (Table 1) describes the consumption of energy to share their data in a particular interval of time with the nodes in the $\mathrm{P} 2 \mathrm{P}$ network. The outcome of the proposed heterogeneous peer node server cycle analyzer using DCDA technique and Node selection strategy is compared with an existing time variant peer heterogeneous P2P network using DTWA.

Figure 5 describes the consumption of energy charged when number of nodes in the network increases to process the appropriate data. In the proposed ELBSHN, the number of the peer nodes increases in the network, then the energy consumption for load balancing factor decreases. The proposed ELBSHN technique is used to efficiently balance the load among the peer nodes without any overflow. Each peer server node utilizes less 
amount of duty cycles to process it, so the consumption of energy for balancing the loads are less. Compared to an existing works like time variant peer heterogeneous P2P network using DTWA, heterogeneous peer node server cycle analyzer and load imbalance factor mechanism, the ELBSHN consumes less energy to perform a load balancing process and the variance is 70$80 \%$ less in the ELBSHN.

The utilization of node for load balancing factor is determined based on the node movement factor in the dynamic P2P systems is illustrated in Table 2.

Figure $\mathbf{6}$ captures the tradeoff between load movement and 99.9th percentile node utilization. Every point on the lower line communicates to the special effects of algorithm with a scrupulous option of load balance time $\mathrm{T}$. One has to give of deciding $\mathrm{T}$ to cooperate among these two metrics in the method which is most suitable for the objective application. But for existing works, for almost all nodes' loads to wait underneath some threshold, a dreadfully minute load harmonizing period $\mathrm{T}$ is employed, so that it is implausible that a node's load increases considerably among periodic poises. This means the existing algorithms to shift considerably more load.

The execution time desired to perform the load balancing factors based on the number of nodes present in the network is depicted in above Table 3.

Figure 7 illustrates the average overall execution time required to perform the load balancing procedures to operate in it. ELBSHN scheme consumes less time to execute the load balancing processes. Since the ELBSHN performed load balancing in two ways, the time taken to distribute and re-allocate the loads for the nodes in the network is less. Rather than using the existing works like time variant peer heterogeneous $\mathrm{P} 2 \mathrm{P}$ network using DTWA, heterogeneous peer node server cycle analyzer using DCDA technique and Node selection strategy for load balancing factors, the ELBSHN achieves better balancing the load in a less interval of time.

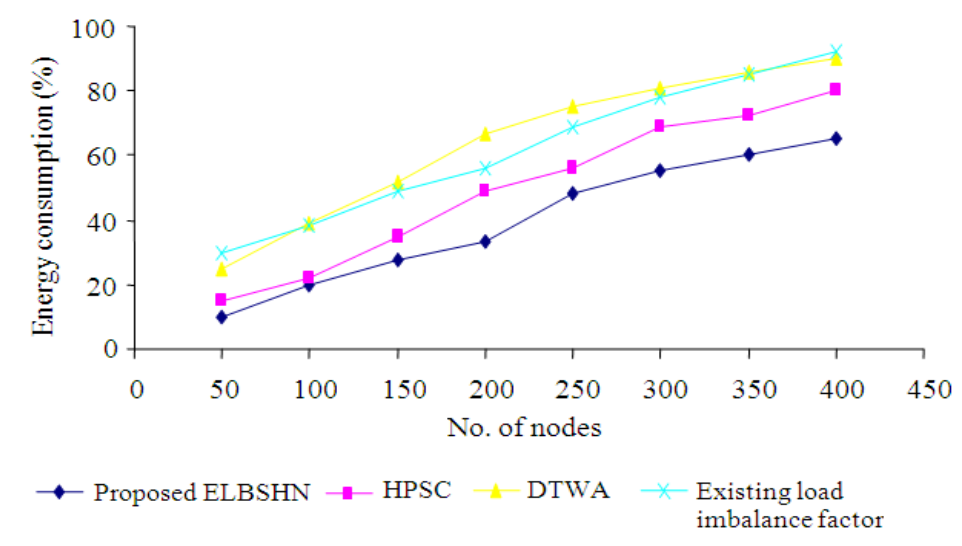

Fig. 5. No. of nodes Vs Energy consumption

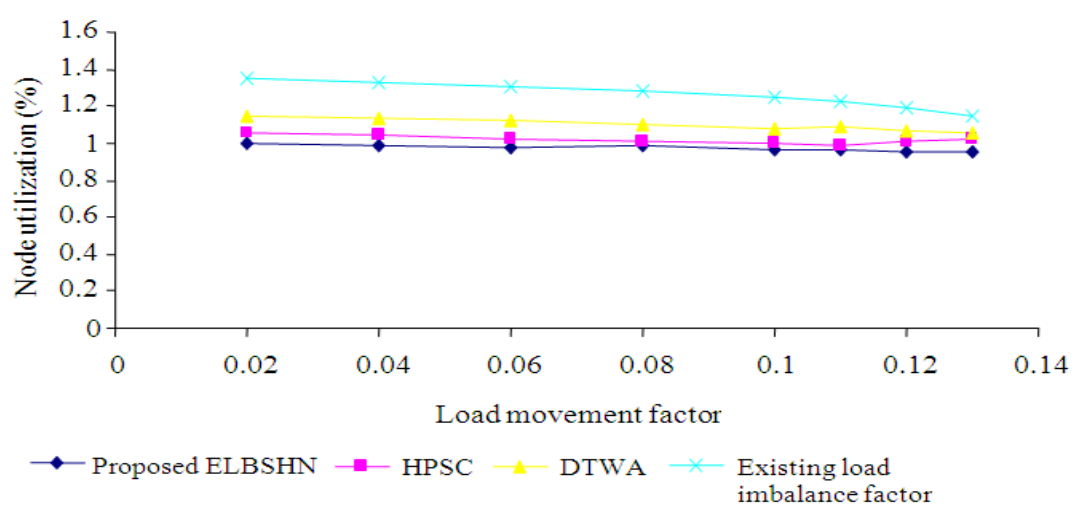

Fig. 6. Load movement factor Vs node utilization 


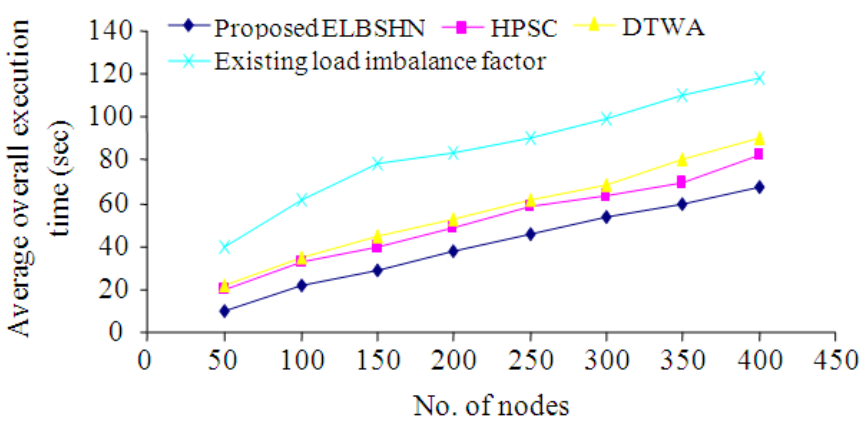

Fig. 7. No. of nodes Vs average overall execution time

Table 1. No. of nodes Vs Energy consumption

\begin{tabular}{llllc}
\hline & \multicolumn{2}{l}{ Energy consumption (\%) } & \\
No. of & Proposed & & & $\begin{array}{l}\text { Existing load } \\
\text { imbalance factor }\end{array}$ \\
\hline nodes & ELBSHN & HPSC & DTWA & 30 \\
100 & 10 & 15 & 25 & 38 \\
150 & 20 & 22 & 39 & 49 \\
200 & 33 & 35 & 52 & 56 \\
250 & 48 & 49 & 67 & 69 \\
300 & 55 & 56 & 75 & 78 \\
350 & 60 & 72 & 81 & 85 \\
400 & 65 & 80 & 90 & 92 \\
\hline
\end{tabular}

Table 2. Load movement factor vs. node utilization

\begin{tabular}{lllll}
\hline & \multicolumn{3}{c}{99.9 th percentile Node utilization } \\
Load move- & Proposed & & \multicolumn{3}{c}{$\begin{array}{l}\text { Existing load } \\
\text { ment factor }\end{array}$} & ELBSHN & HPSC & DTWA & imbalance factor \\
\hline 0.02 & 1.00 & 1.05 & 1.15 & 1.35 \\
0.04 & 0.99 & 1.04 & 1.13 & 1.33 \\
0.06 & 0.98 & 1.02 & 1.12 & 1.30 \\
0.08 & 0.99 & 1.01 & 1.10 & 1.28 \\
0.10 & 0.97 & 1.00 & 1.08 & 1.25 \\
0.11 & 0.96 & 0.99 & 1.09 & 1.23 \\
0.12 & 0.95 & 1.01 & 1.07 & 1.19 \\
0.13 & 0.95 & 1.02 & 1.05 & 1.15 \\
\hline
\end{tabular}

Table 3. No. of nodes vs. average overall execution

\begin{tabular}{|c|c|c|c|c|}
\hline \multirow[b]{2}{*}{$\begin{array}{l}\text { No. of } \\
\text { nodes }\end{array}$} & \multicolumn{4}{|c|}{ Average overall execution time (sec) } \\
\hline & $\begin{array}{l}\text { Proposed } \\
\text { ELBSHN }\end{array}$ & HPSC & DTWA & $\begin{array}{l}\text { Existing load } \\
\text { imbalance factor }\end{array}$ \\
\hline 50 & 10 & 20 & 22 & 40 \\
\hline 100 & 22 & 33 & 35 & 62 \\
\hline 150 & 29 & 40 & 45 & 78 \\
\hline 200 & 38 & 49 & 53 & 83 \\
\hline 250 & 46 & 59 & 62 & 90 \\
\hline 300 & 54 & 64 & 69 & 99 \\
\hline 350 & 60 & 70 & 80 & 110 \\
\hline 400 & 68 & 82 & 90 & 118 \\
\hline
\end{tabular}

Finally it is being observed that the ELBSHN performs effective load balancing in a heterogeneous network. An enhanced load balancing scheme provide a desirable, flexible load balancing and high system capacity in a heterogeneous P2P network. Simulation results show that the ELBSHN scheme improves system capacity and resource utilization compared with the conventional time variant peer heterogeneous P2P network using DTWA, heterogeneous peer node server cycle analyzer using DCDA technique and Node selection strategy for load balancing factors.

\section{CONCLUSION}

Presented a novel and enhanced load balancing scheme for heterogeneous P2P systems. Examine the concern vigilantly and form out that the load balancing presentation in existing systems which is greatly exaggerated by system properties for instance the peer heterogeneity, P2P overlie network topology. In resolution, all these concerns are well considered to produce better load balance choices. Initially, generate an efficient method to preserve the file access record information and employ this information to envisage the future file access actions. Secondly, by employing this information, which can more precisely divide the workload when a novel peer unites the system; then, by employing this information, also generate enhanced load reorganization choices by adapting the sizes of nodes capacities.

The load redeployment is only achieved when it is needed; the persuaded overhead crisis is reduced. With this approach, load can be fine evenhanded on numerous peers, the user alleged regaining overhead is condensed and the bandwidth utilization is reduced as well. Simulation results show that the ELBSHN scheme improves system capacity and resource utilization compared with the conventional time 
variant peer heterogeneous P2P network using DTWA, heterogeneous peer node server cycle analyzer using DCDA technique and Node selection strategy for load balancing factors.

\section{REFERENCES}

Alakeel, A.M., 2012. A fuzzy dynamic load balancing algorithm for homogenous distributed systems. World Acad. Sci., Eng. Technol., 61: 30-33.

Hsiao, H.C., H. Liao, S.T. Chen and K.C. Huang, 2011. Load Balance with imperfect information in structured peer-to-peer systems. IEEE Trans. Parallel Distrib. Syst., 22: 634-649. DOI: 10.1109/TPDS.2010.105

Hu, Q., Y. Yu, Z. Cai, J.E. Womack and Y. Song, 2010. Mobile association in a heterogeneous network. Proceedings of the IEEE International Conference on Communications, May 23-27, IEEE Xplore Press, Cape Town, pp: 1-6. DOI: 10.1109/ICC.2010.5502817

Lee, S., W. Su, S. Batalama and J.D. Matyjias, 2010. Cooperative decode-and-forward ARQ relaying: Performance analysis and power optimization. IEEE Trans. Wireless Commun., 9: 2632-2642. DOI: 10.1109/TWC.2010.062310.091554
Salem, M., A. Adinoyi, M. Rahman, H. Yanikomeroglu and D. Falconer et al., 2010. Fairness-aware radio resource management in downlink OFDMA cellular relay networks. IEEE Trans. Wireless Commun., 9: 1628-1639. DOI: 10.1109/TWC.2010.05.081548

Son, K., S. Chong and G. Veciana, 2009. Dynamic association for load balancing and interference avoidance in multi-cell networks. IEEE Trans. Wireless Commun., 8: 3566-3576. DOI: 10.1109/TWC.2009.071140

Tang, Y., S. Zhou and J. Xu, 2010. LIGHT: A queryefficient yet low-maintenance indexing scheme over DHTs. IEEE Trans. Knowl. Data Eng., 22: 59-75. DOI: 10.1109/TKDE.2009.47

Yang, Y., H. Hu, J. Xu and G. Mao, 2009. Relay technologies for WiMax and LTE-advanced mobile systems. IEEE Commun. Mag., 47: 100-105. DOI: 10.1109/MCOM.2009.5273815 